\title{
Piggyback IOL implantation: indication, surgical techniques and complications
}

\begin{abstract}
Purpose: Piggyback IOL implantation can be performed during cataract surgery or refractive lens exchange and IOL insertion (primary piggyback implantation) to manage high IOL power or as a secondary procedure following the initial IOL implantation (secondary piggyback implantation) to manage refractive surprise. To report visual outcome after primary and secondary piggyback IOL implantation procedure.

Methods: Implantation of piggyback IOLs in ciliary sulcus were done as primary or secondary manner to correct refractive surprise. For minus (myopic) refractions, multiply the spherical equivalent by 1.2 to determine the piggyback IOL power. For plus (hyperopic) refractions, multiply the spherical equivalent by 1.5 to determine the piggyback IOL power.

Results: All cases achieved excellent visual outcome with no complications as interleticular opacity, glaucoma, UGH syndrome.

Conclusion: Primary piggyback IOL implantation provides adequate IOL power to a patient with extreme hyperopia. Secondary piggyback IOL implantation is helpful in pseudophakic patients to correct residual refractive error. Psedophakic supplementary IOLs (Rayner Sulcoflex) are now available for secondary piggyback IOL implantation. Combinations of monofocal, toric and multifocal IOL implantation in piggyback manner is a viable and useful option for patients needing very high IOL power.
\end{abstract}

Volume 8 Issue 2 - 2018

\author{
Suresh K Pandey, ${ }^{1,2,3}$ Vidushi Sharma \\ 'SuVi Eye Institute \& Lasik Laser Centre, India \\ ${ }^{2} J o h n$ a Moran Eye Center, University of Utah, USA \\ ${ }^{3}$ University of Sydney, Australia
}

Correspondence: Suresh K Pandey, SuVi Eye Institute \& Lasik Laser Centre, C I 3 Talwandi, KOTA, Rajasthan, 324 005, India, Tel +91 935 |4| 2449, Email suvieye@gmail.com

Received: December 30, 2017| Published: April 30, 2018

\section{Introduction}

Technique of piggyback (back-to-back) intraocular (IOL) implantation (also termed as poly-pseudophakia) was first introduced by Dr. Johhny L Gaytonin 1993. The first report of the placement of two IOL implants in piggyback manner in a highly hyperopic eye was by Jim P. Gills, MD in 1993.Placing an additional IOL in piggyback manner is an effective way to correct a residual refractive error, particularly high degrees of refractive surprise. Review of literature revealed increasing use of piggyback implantation for correction of residual refractive errors as well as few case report of complication such as interlenticular opacity and methods to prevent such complications. ${ }^{1-7}$ Pseudophakic supplementary IOLs (Sulcoflex monofocal, toric and multifocal IOLs) are now available.

\section{Types of piggyback IOL implantation}

i. Primary Polypseudophakia: High hyperopia needing high IOL power is cataract cases with very short axial lenghth (e.g. nanophthalmos/micro-ophthalmos).

ii. Secondary Polypseudophakia: To manage postoperative refractive surprise.

\section{Work up \& preoperative preparation for piggyback IOL implantation}

A meticulous preoperative preparation and workup is essential before planning primary or secondary piggyback IOL implantation. Preoperative workup includes thorough anterior and posterior segment examination with an emphasis on intraocular pressure, corneal endothelium cell count, anterior chamber depth, etc. For secondary piggyback IOL implantation, the first IOL should be well centered in the capsular bag. A Preoperative YAG Laser peripheral iridectomy (or surgical peripheral iridectomy) is usually not needed in most of cases, however this can be done in cases with shallow anterior chamber or in some cases predisposed to glaucoma.

\section{Piggyback IOL power calculations}

\section{A. Primary piggyback IOL implantation}

Dr Warren Hills described six steps for IOL Power Calculation for piggyback IOL; Details can be seen from website: http://www.doctorhill.com/iol-main/polypseudophakia_calculations.htm)

1. Step I. Measure the axial length as accurately as possible.

2. Step II. Calculate the total IOL power needed at the plane of the capsular bag.

3. Step III. Calculate the residual IOL power.

4. Step IV: Determine the power adjustment for the anterior (ciliary sulcus) lens.

5. Step V: Calculate the power of the anterior IOL.

6. Step VI: Select the appropriate polypseudophakia lens pair.

\section{B. Secondary piggyback IOL implantation}

This is based on residual refractive error. For Myopia: Spherical equivalent multiplied by 1.15

For Hyperopia: Spherical equivalent multiplied by 1.25 
More advanced IOL Power calculation: Holladay consultant IOL software \& surgical outcome assessment details are available on website www.hicsoap.com This is based on residual refractive error, $\mathrm{K}$ reading and anterior chamber depth.

\section{Surgical techniques for piggyback IOL implantation}

For piggyback IOL implantation, we prefer to implant threepiece IOL in the ciliary sulcus. The surgeon should never implant the square-edged hydrophobic acrylic IOL in the ciliary sulcus to minimize the iris chaffing and glaucoma. In case of high hyperopia with short anterior chamber depth, care should be taken to minimize the endothelial cell loss by using chondroitin sulfate based dispersive ophthalmic viscoelastic device (OVD) (DuoVisc or Viscoat- Alcon Laboratories). Care should be taken to remove the OVD from the anterior chamber after surgery to minimize post-operative pressure spike.

\section{Contraindications of piggyback IOL implantation}

Piggyback IOL implantation should not be attempted in cases of poor endothelial cell count, cases of uveitis and glaucoma patients.

\section{Authors experience and case reports of piggyback IOL implantation}

During past 5 years, we have performed piggyback IOLs in more than 28 cases. Selected cases are discussed here.

\section{Case I: Refractive lens exchange and primary piggyback IOL implantation for high hyperopia}

Fifty year old male patient presented to us with complains of poor vision without glasses. His refraction was $+8 \mathrm{D} \mathrm{Sph} /+1.75 \mathrm{D}$ Cyl at 20 degree; addition plus 2.50 (Right eye). Patient was interested for freedom from near and distance glasses. Preoperative keratometry revealed 1.25 Diopter of corneal astigmatism. IOL power calculation for right eye was plus 34.50 Diopter. During the time of surgery, multifocal and toric IOL were available only till 30 Diopters. We planned for primary piggyback IOL implantation of mutifocal IOL (15.0 D, Acridiff- Care Group, India) and toric (Acrioltoric 19.50 Diopter, Care Group, India). The reference marking was done in sitting position on slit lamp and axis marking was performed in the operating room. (Figure 1) Side-port incision was made and this is followed by injection of OVD (Alcon Viscoat) in the anterior chamber to coat the corneal endothelium. Main incision was created at steep axis ( 20 degree). This was followed by well centered $5 \mathrm{~mm}$ anterior capsulorhexis that is crucial for perfect centration of toric and multifocal IOLs. Cortico-cleaving hydro-dissection was performed and phaco-aspiration was done using low phaco power and fluidics parameters. This was followed by bimanual irrigation and aspiration of cortical matter.

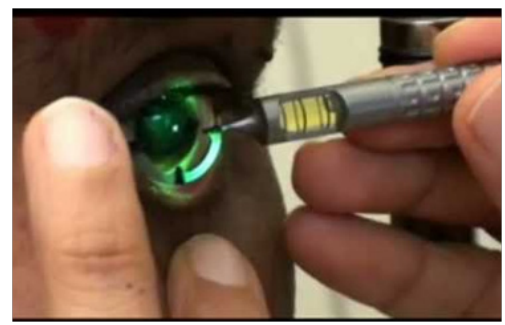

Figure IA Reference Marking on slit lamp.

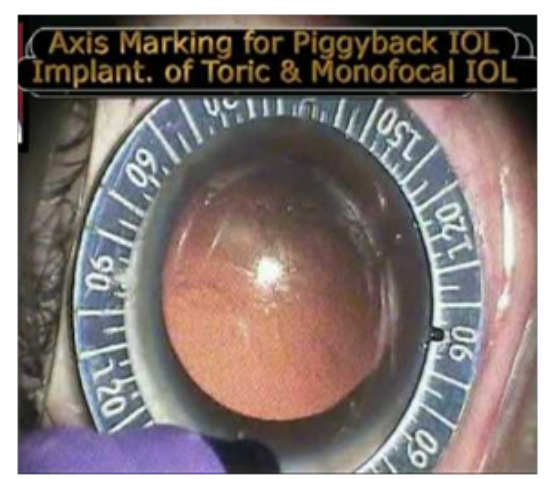

Figure IB Axis marking on OT Table.

Step I: Implantation of Toric IOL: After filling the capsular bag with OVD ( $2 \%$ Hydroxyprolpyl methyl cellulose HPMC), toric IOL (19.5 D, Acriol Toric IOL- Care Group, India) implantation was done in the capsular bag using an injector. The IOL haptics were dialed in the capsular bag and OVD was removed behind the IOL using bimanual irrigation and aspiration. Final alignment of the toric IOL was done along the astigmatic axis (20 degree).

Step II: Implantation of Multifocal IOL: We then implanted multifocal IOL (15.0 Diopter Acridiff multifocal IOL- Care Group India) in the capsular bag. Standard practice is to implant one IOL in the capsular bag and another IOL in the ciliary sulcus, however, in this case we implanted both IOLs (Acrioltoric and Acridiff multifocal IOL) in the capsular bag as precise centration and position in the capsular bag is must for refractive outcome. OVD was removed between the IOL and from the anterior chamber using bimanual irrigation and aspiration. (Figure 2) We performed YAG Laser assisted enlargement of anterior capsulorhexis opening was done after 8 weeks time to prevent occurrence of interlenticular opacification. Postoperative visual outcome was $6 / 6$ and N5 (unaided) and the patient was very satisfied with the visual outcome.

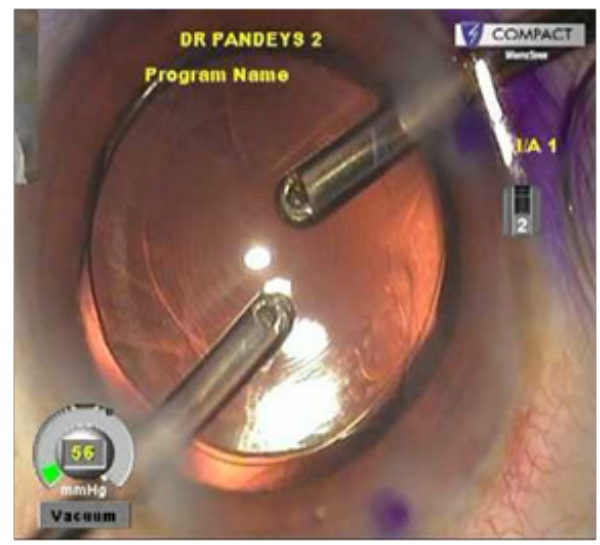

Figure 2 Removal of OVD from capsular bag and anterior chamber after piggyback IOL Implantation.

\section{Case 2: primary piggyback IOL implantation for developmental cataract}

We used combination of monofocal and multifocal IOL was used to obtain excellent visual outcome in 12 years old child with developmental cataract (right eye). Side-port incision was created 
and this was followed by injection of dispersive OVD in the anterior chamber. Main incision was made at steep axis. This was followed by creation of circular anterior capsulorhexis of approximately 5.0 $\mathrm{mm}$ in size. Cortico-cleaving hydro-dissection was done and phacoaspiration of soft lens matter using bimanual irrigation and aspiration. Cortical matter was removed from capsular bag and implantation of multifocal IOL (+29.0 Diopter Acridiff Care Group, India) was done in the capsular bag. This was followed by implantation of monofocal IOL (+3.5 Diopter Flexifold IOL, Care Group, India) in the ciliary sulcus. The OVD was removed between the IOL and from the capsular bag. Post-operative visual outcome was excellent and patient regained visual outcome of 20/20 and N6 unaided.

\section{Case 3: secondary piggyback iol implantation of rayner sulcoflex IOL}

A 14 years old child of developmental cataract underwent cataract surgery with toric IOL implantation in the right eye. Postoperatively the vision improved to $6 / 6$ unaided. The child and his parents were keen to have good near vision without glasses. We plan secondary implantation of Sulcoflex multifocal IOL (Rayner UK) to achieve good refractive outcome. The side-port incision was made and this was followed by injection of OVD in the anterior chamber. This was followed by creation of main incision at steep axis. We loaded Sulcoflex multifocal IOL (distance power zero with plus 3.5 D near add) in the cartridge and IOL implantation was done in the cilary sulcus. Sulcoflex IOL is specially designed to be implantation in piggyback manner in the ciliary sulcus as it has round edge and undulating haptics that minimize the chanced of iris chaffing. The alignment of haptics of secondary piggyback IOL haptics can be done in the same direction of primary IOL haptics or they can be aligned 90 degree to the primary IOL haptics. In this case, care was taken not to disturb the toric IOL alignment. The OVD was removed between the IOL optics and from the anterior chamber. This combination of placing one IOL the capsular bag and another one in the cilary sulcus (with different IOL combination) minimize the chances of interlenticular opacification. The child achieved excellent distance and near vision (20/20 and N5) unaided.

\section{Complications of piggyback IOL procedure}

Complications reported with piggyback IOL implantation is interlenticular opacification (also termed as inter-pseudophakos opacification or red rock syndrome). This complication was first reported by Gayton et al., ${ }^{7}$ with hydrophobic IOL implantation (Alcon AcrySof IOL) implanted in the capsular bag with small rhexis. ${ }^{8}$

\section{Discussion}

Piggyback IOLs can use during the primary implantation or as secondary procedure to correct refractive surprise. First reported by Johnny L Gayton, M.D of Warner Robins, GA, USA, in 1993, piggyback placement of an IOL can be a successful strategy for correcting residual refractive error. With the recent introduction of Sulcoflex IOL, the use of piggyback implantation for correction of residual refractive errors appears to be increasing in popularity. Placing an additional IOL in the capsular bag/cilary sulcus is an effective way to correct a residual refractive error, particularly high degrees of refractive surprise. With the original IOL placed in the capsular bag, the piggyback IOL is implanted in the ciliary sulcus to avoid inter-lenticular opacification (also termed as inter-pseudophakos opacification or red rock syndrome). Piggyback IOL implantation can be performed during cataract surgery or refractive lens exchange and IOL insertion (primary piggyback implantation) to manage high IOL power or as a secondary procedure following the initial IOL implantation (secondary piggyback implantation) to manage refractive surprise. IOL Power calculation for piggyback IOLs is based on residual refractive error and can achieve highly accurate outcomes. For minus (myopic) refractions, multiply the spherical equivalent by 1.2 to determine the piggyback IOL power. For plus (hyperopic) refractions, multiply the spherical equivalent by 1.5 to determine the piggyback IOL power. Postoperative complications, of the piggyback IOL procedure include interlenticular opacification (ILO), uveitis and glaucoma and these can be avoided with proper preoperative planning, IOL selection, surgical procedure and meticulous follow-up.

Primary piggyback IOL implantation provides adequate IOL power to a patient with extreme hyperopia. Secondary piggyback IOL implantation is helpful in pseudophakic patients to correct residual refractive error. Psedophakic supplementary IOLs (Rayner Sulcoflex) are now available for secondary piggyback IOL implantation. A combination of monofocal, toric and multifocal IOL implantation in piggyback manner is a viable and useful option for patients needing very high IOL power.

Pearls for Piggyback Intraocular Lens Implantation Dr Suresh K Pandey Dr Vidushi Sharma Kota

\section{Acknowledgements}

None.

\section{Conflict of interest}

The author declares that there is no conflict of interest.

\section{References}

1. Gayton JL, Sanders VN. Implanting two posterior chamber intraocular lenses in a case of microphthalmos. $J$ Cataract Refract Surg. 1993;19(6):776-777.

2. Rubenstein JB. Piggyback IOLs for residual refractive error after cataract surgery. Cataract and Refractive Surgery Today. 2012;38:155-162.

3. Holladay JT, Gills JP, Leidlein J, et al. Achieving emmetropia in extremely short eyes with two piggyback posterior chamber intraocular lenses. Ophthalmology. 1996;103(7):1118-1123.

4. Shugar JK, Lewis C, Lee A. Implantation of multiple foldable acrylic posterior chamber lenses in the capsular bag for high hyperopia. J Cataract Refract Surg. 1996;22(Suppl 2):1368-1372.

5. Shugar JK, Schwartz T. InterpseudophakosElschnig pearls associated with late hyperopic shift: A complication of piggyback posterior chamber intraocular lens implantation. J Cataract Refract Surg. 1999;25(6):863867.

6. Shugar JK, Keeler S. Inter-pseudophakos intraocular lens surface opacification as a late complication of piggyback acrylic posterior chamber intraocular lens implantation. J Cataract Refract Surg. 2000;26(3):448455 .

7. Gayton JL, Apple DJ, Peng Q, et al. Interlenticular opacification: A clinicopathological correlation of a new complication of piggyback posterior chamber intraocular lenses. J Cataract Refract Surg. 2000;26(3):330-336.

8. Werner L, Shugar JK, Apple DJ, et al. Opacification of piggyback IOLs associated with an amorphous material attached to interlenticular surfaces. J Cataract Refract Surg. 2000;26(11):1612-1619. 